\title{
AUDIT ON ADHERENCE TO 2010 EUROPEAN CONSENSUS GUIDELINES ON THE MANAGEMENT OF NEONATAL RESPIRATORY DISTRESS SYNDROME IN PRETERM INFANTS
}

\author{
K. Tanney, D. Sweet \\ Neonataology, Royal Maternity Hopsital, Belfast, UK
}

Background and aims: Controversies exist around perinatal management of RDS. Antenatal steroids and surfactant replacement therapy are clearly important, but issues of timing of interventions still cause debate. Recently updated Consensus Guidelines outline evidence from Cochrane review and medical literature advocating practices to minimize consequences of RDS. The aim of this study was to identify areas where guidelines are adhered to, and those to be targeted for improvement.

Methods: We carried out a retrospective observational study of the first 20 infants born $<28$ weeks' gestation in our unit in 2010. We assessed management in areas of prenatal care, delivery room stabilisation, surfactant therapy and respiratory support.

Results: In $\geq 75 \%$ of cases, infants were managed as per guidelines in aspects of: delivery centre, documentation of Neopuff and Neowrap bag use at resuscitation, early surfactant prophylaxis and CPAP pressures $\geq 6$, use of SIMV and Caffeine to facilitate weaning (with gas $\mathrm{pH}>7.22$ ), extubation to CPAP / NIPPV, appropriate antibiotic use while excluding sepsis, and early introduction of TPN and enteral feeds. Complete adherence was seen in hypocapnia avoidance, minimising mechanical ventilation duration, incubator humidification and temperature maintenance. Documentation of oxygen titration and maternal antibiotics for PROM show room for improvement (25\%).

Conclusions: In this tertiary centre, many aspects of care adhere to European guidelines, while others should be improved upon as evidence expands and practices evolve. We continue to strive to meet guidelines in this challenging area, and will re-audit to assess improvement in standards of care. 